\title{
Reviewing Treatment Options for Advanced Renal Cell Carcinoma: Is There Still a Place for Tyrosine Kinase Inhibitor (TKI) Monotherapy?
}

\author{
Mário Fontes-Sousa (D) · Helena Magalhães (D) - Alicia Oliveira (D) • \\ Filipa Carneiro · Filipa Palma dos Reis (D) · Pedro Silvestre Madeira (D) • \\ Sara Meireles
}

Received: October 7, 2021 / Accepted: November 26, 2021 / Published online: January 13, 2022

(C) The Author(s) 2022

\begin{abstract}
Renal cell carcinoma (RCC) comprises a highly heterogeneous group of kidney tumours built upon distinct genetic- and epigenetic-driven mechanisms and molecular pathways. Therefore, responsiveness to treatment is considerably variable across patients, adding an extra layer of complexity to the already challenging therapeutic decision process. The last decade brought an unprecedented shift in the medical approach to advanced or metastatic RCC; in fact, immunotherapy-based combinations have significantly transformed the therapeutic arsenal and clinical outcomes of these patients. These
\end{abstract}

M. Fontes-Sousa ( $\square)$

Hospital CUF Tejo, Av. 24 Julho, 171A, 1350-352

Lisbon, Portugal

e-mail: mario.fontes.sousa@cuf.pt

\section{H. Magalhães}

Hospital Pedro Hispano (Unidade Local de Saúde de Matosinhos), Rua Dr. Eduardo Torres, 4464-513

Senhora da Hora, Portugal

A. Oliveira

Hospital do Espírito Santo de Évora, Largo do Sr. da Pobreza, 7000-811 Évora, Portugal

\section{F. Carneiro}

Medical oncology department, Instituto Português de Oncologia Do Porto, Rua Dr. António Bernardino de Almeida 865, 4200-072 Porto, Portugal

e-mail: ana.carneiro@ipoporto.min-saude.pt strategies were quickly adopted by international guidelines committees as the new standards of care. However, this enhanced efficacy comes at the expense of tolerability, with a predictable negative impact on patients' quality of life. Moreover, subgroup and post hoc analyses of the major clinical trials have shown that not all patients benefit equally from these innovative approaches. In this context, a group of experts on kidney cancer met and discussed the state of the art in the field, with a special emphasis on the appropriateness of using monotherapy with an anti-angiogenesis tyrosine kinase inhibitor (TKI) to treat specific subgroups of patients with RCC. This article reviews the main topics that were

\section{F. P. dos Reis}

Hospital de Santo António Dos Capuchos (Centro Hospitalar Universitário de Lisboa Central),

Alameda Santo António Dos Capuchos, 1169-050 Lisbon, Portugal

\section{P. S. Madeira}

Instituto Português de Oncologia de Coimbra, Av. Prof. Dr. Bissaya Barreto No. 98, 3000-075 Coimbra, Portugal

\section{S. Meireles}

Hospital de São João (Centro Hospitalar

Universitário de São João), Alameda Prof. Hernâni Monteiro, 4200-319 Porto, Portugal 
considered to be pertinent for that discussion and establishes the profile of patients for whom TKI monotherapy remains a sensible frontline option by avoiding overtreatment and an unnecessary exposure to treatment-related toxicity.

Keywords: Advanced or metastatic renal cell carcinoma (mRCC); Immune checkpoint inhibitor (ICI); Monotherapy; Tyrosine kinase inhibitor (TKI)

\section{Key Summary Points}

The treatment of advanced or metastatic renal cell carcinoma (mRCC) has changed dramatically over the last decades: an initially unspecific immune approach has evolved into a targeted strategy, which more recently incorporated the simultaneous use of two agents (as opposed to the more traditional monotherapy).

These combinations involve either two immune checkpoint inhibitors (ICIs) or an ICI associated with an antiangiogenesis drug (usually a tyrosine kinase inhibitor [TKI] targeted at the vascular endothelial growth factor) and were shown to significantly extend survival in a wide range of patients with mRCC.

However, one should take into consideration that the use of two drugsinstead of a single agent-often impacts treatment tolerability and patients' quality of life, while possibly limiting the range of therapeutic weapons available for subsequent therapeutic lines.

Additionally, not all patients benefit equally from combination treatments; whereas these strategies have a highly significant effect in patients with an intermediate or poor prognosis, their advantages are limited in patients with a favourable one.
A group of kidney cancer experts met in a series of virtual meetings to review the evidence related to the aforementioned points and to discuss the pertinence of TKI monotherapy in selected patients with mRCC: the evidence considered to be relevant for that discussion is described in this article, as are the main conclusions reached by the panel.

\section{INTRODUCTION}

Renal cell carcinoma (RCC) is a heterogeneous group of malignancies that account for $2 \%$ of global cancer diagnoses and deaths [1]. Associated with several risk factors that are characteristic of so-called modern societies, RCC incidence has been rapidly increasing in the developed world, being now the seventh most common neoplasm in these regions $[1,2]$. Of note, RCC has the highest fatality rate among urological neoplasms; whereas the overall 5 -year survival rate is $76 \%$, this value drops dramatically to $12 \%$ in patients with stage IV disease [1]. Also, approximately $30 \%$ of patients newly diagnosed with RCC have metastatic disease, while $20-50 \%$ of patients treated for localised disease will eventually relapse and progress to the metastatic stage $[1,3]$. Importantly, adjuvant therapeutic alternatives are yet to be approved for this setting in Europe.

RCC incidence and mortality rates vary widely according to its subtype; in fact, RCC comprises a group of independent histological entities, which are characterised by distinctive genetic and epigenetic alterations and molecular pathways, and as such, they respond to systemic therapy $[4,5]$. The most common RCC subtype is clear cell, which accounts for $70-75 \%$ of all cases. From a genetic standpoint, this subtype is characterised by the loss of the short arm of chromosome 3, which encodes the tumour suppression gene VHL (von Hippel-Lindau) [4, 6, 7]. The other RCC forms are generally grouped under an umbrella term: the non-clear cell subtypes. Among these, the papillary variant has the highest incidence, comprising $15 \%$ of all kidney cancers $[4,6,8]$. 
Chromophobe RCC, which occurs in $5-10 \%$ of all cases, has a typically indolent course, although it is particularly hard to treat once it has metastasized $[4,6,7]$. Collecting duct carcinoma and renal medullary carcinoma account for less than 5\% of all RCC cases. These rare subtypes are often aggressive, being generally resistant to most systemic therapy options available to date $[6,7]$. Finally, about $5 \%$ of all RCC cases have sarcomatoid features; sarcomatoid RCC is usually symptomatic and highly aggressive, and its outcomes tend to be worse than those of non-sarcomatoid cases $[9,10]$.

Even among the clear cell subtype, advanced or metastatic RCC (mRCC) is known to be highly heterogeneous in terms of clinical progression and treatment outcomes. For that reason, a few prognostic risk models have been developed, of which the two most commonly used are the Memorial Sloan Kettering Cancer Center model (MSKCC) and the International mRCC Database Consortium model (IMDC). The MSKCC model was based on the retrospective analysis of 463 patients with mRCC treated with interferon- $\alpha$, in whom the authors were able to identify five risk factors: low Karnofsky performance status (less than $80 \%$ ), high serum lactate dehydrogenase (above 1.5 times the upper limit of normal), low haemoglobin (below the lower limit of normal), high corrected serum calcium (above $10 \mathrm{mg} / \mathrm{dL}$ ) and a short time-period between the initial diagnosis and systemic therapy onset (less than 1 year) [11]. Patients were then stratified into three categories according to the number of risk factors present: those without risk factors were classified as of favourable risk; those with one or two risk factors were classified as of intermediate risk; and those with three or more risk factors were classified as poor risk. Accordingly, the median overall survival (OS) was 30, 14 and 5 months in each of these groups, respectively [11]. IMDC was developed a few years later, being based on a retrospective analysis of 645 patients with mRCC treated with tumour vasculaturetargeted therapy. The IMDC authors retained four MSKCC risk factors-low Karnofsky performance status (less than 80\%), high corrected serum calcium (above the upper limit of normal), low haemoglobin (below the lower limit of normal) and a short time-period between initial diagnosis and systemic therapy onset (less than 1 year)—and added high neutrophil and platelet counts (above the upper limit of normal) [12]. After 2 years, $75 \%$ of the favourable-risk patients were still alive, as were $53 \%$ of the intermediaterisk patients and $7 \%$ of the poor-risk patients [12]. Afterwards, several adjustments were made to these models, either to validate them in a salvage setting $[13,14]$ or to include other significant factors, such as metastasis location [15]. Still, despite a number of acknowledged limitations, the classical version of these prognostic models remains commonly used both in clinical trials and during routine clinical practice.

The management of advanced or mRCC has changed dramatically over the past 30 years. Initially based on a non-specific immune approach (high-dose interleukin-2 [IL-2] and interferon- $\alpha$ ), this strategy evolved to target the tumour vasculature, intracellular oncogenic pathways and the immune system signalling cascade. The new agents added to the mRCC therapeutic armamentarium include vascular endothelial growth factor (VEGF)-targeted molecules, inhibitors of the mechanistic target of rapamycin (mTOR), and novel immune checkpoint inhibitors (ICIs) [3, 6]. Recently, international guidelines have suggested a combination of two of these agents (ICI/ICI or ICI/ VEGF-targeted agent) as the best strategy to manage clear cell mRCC [16-18]. However, despite the undeniable benefits of these combination strategies-which were demonstrated in a number of clinical trials-they also represent enhanced toxicity when compared with monotherapy, with a predictable negative impact on patients' quality of life (QoL), and likely limit the choice of subsequent therapeutic lines. Although this may be a result of the specific anti-VEGF tyrosine kinase inhibitor (TKI) used (e.g. pazopanib or sunitinib), the possible high grade of toxicity in a metastatic setting should be taken into careful consideration. However, whether combination treatment is always the most sensible option to treat mRCC is a question yet to be answered. Whereas the efficacy of any treatment is a key factor in the therapeutic decision-making process, avoiding overtreatment and unnecessary toxicity should also be carefully considered. In 
this context, a group of Portuguese experts participated in a series of virtual meetings held between February and April 2021, which were aimed at reviewing the clinical evidence concerning the frontline treatment of mRCC, as well as the profile of patients for whom TKI monotherapy is still the best available therapy. The topics considered to be relevant to this discussion are reviewed hereafter, as are the main conclusions reached by this panel of experts. This article is based on previously conducted studies and does not contain any new studies with human participants or animals performed by any of the authors.

\section{THE THERAPEUTIC LANDSCAPE OF MRCC}

The first agents to be approved for mRCC treatment were interferon- $\alpha$ and high-dose IL-2. However, despite inducing highly durable responses in a limited number of patients, the efficacy of these drugs was considerably low (the reported response rates were $12 \%$ for interferon$\alpha$ and $15 \%$ for IL-2), and their toxicity was rather high (particularly that of IL-2) [5, 6, 19]. As such, they were later replaced by targeted approaches directed at either the endothelium of the tumour vasculature (anti-VEGF drugs) or at the tumour's oncogenic pathways (mTOR inhibitors). Anti-VEGF drugs include orally available TKIs targeting circulating VEGF itself or its receptors (axitinib, cabozantinib, lenvatinib, pazopanib, sorafenib, sunitinib and tivozanib), as well as an intravenously administered anti-VEGF antibody (bevacizumab combined with interferon alfa-2a), whereas mTOR inhibitors include temsirolimus and everolimus $[6,19]$. All of these agents have shown a progression-free survival (PFS) benefit over existing alternatives and have been extensively reviewed elsewhere [20-22].

Lately, there has been a resurgence of interest in immunotherapy to treat patients with mRCC; contrary to the initial non-specific approach, the most recent immunotherapybased strategies successfully target certain immune checkpoints involved in peripheral tolerance. Nivolumab, an anti-programmed cell death 1 (PD-1) antibody that selectively blocks the interaction between PD-1 and its ligand (PD-L1), was the first ICI approved in this setting. When compared with everolimus in previously treated patients, nivolumab was shown to significantly extend OS (25.0 vs 19.6 months; hazard ratio [HR] $0.73 ; 98.5 \%$ confidence interval (CI) $0.57-0.93 ; p=0.002)$ and to elicit a higher objective response rate (ORR 25\% vs 5\%; odds ratio $[\mathrm{OR}]$ 5.98; $95 \%$ CI $3.68-9.72$; $p<0.001$ ) [23].

Finally, a major paradigm shift took place in the mRCC therapeutic armamentarium as dualagent combinations were introduced and, in many cases, replaced the TKI monotherapy strategy as the standard of care (SOC). One of these combinations involves the concerted action of two ICIs: nivolumab + ipilimumab (an anti-CTL4 [cytotoxic T-lymphocyte-associated protein 4] antibody) [24-26]. Still, the majority of these double treatments consist of the combination of an ICI with a VEGF-targeted drug: avelumab + axitinib [27], atezolizumab + bevacizumab [28], pembrolizumab + lenvatinib [29], pembrolizumab + axitinib [30, 31] and nivolumab + cabozantinib [32]. The rationale behind this strategy is the simultaneous attack of two pivotal features of the RCC tumour: angiogenesis and immunogenic regulation. Indeed, by activating the immune system and suppressing vasculature signalling in the tumour microenvironment, these combination strategies should, in theory, overcome the limitations of each drug individually, leading to a durable immunotherapy-induced response sustained by an effective modulation of the tumour microenvironment [33, 34].

\section{International Guideline Recommended Treatment Strategies}

Facing this rapidly evolving therapeutic landscape, the international guidelines were adapted to consider the new treatment combinations. Some of these combinations are promising, but are not yet recommended as they have failed to demonstrate a significant OS signal (e.g. avelumab + axitinib and 
axitinib + atezolizumab). However, other combinations have been already considered as the new SOC in mRCC. In fact, in an eUpdate published in September 2021, the European Society for Medical Oncology (ESMO) recommends the use of pembrolizumab + lenvatinib, pembrolizumab + axitinib or cabozantinib + nivolumab as first-line treatment for all patients with clear cell mRCC, irrespective of their IMDC risk group (all recommendations, level I, A; ESMO-MCBS v1.1, score 4) [16]. In patients with an IMDC favourable-risk prognosis, sunitinib or pazopanib are potential alternatives to PD-1 inhibitor-based combination treatment because of a lack of clear superiority of PD-1 inhibitor-based combinations over sunitinib in these patients, and the similar effectiveness of sunitinib and pazopanib in the COMPARZ study [35] (level I, B) [16].

The combinations of pembrolizumab + axitinib, cabozantinib + nivolumab and pembrolizumab + lenvatinib are also recommended as a preferred first-line approach for all patients with RCC according to the latest update of the National Comprehensive Cancer Network guidelines (with category 1 as the evidence level) [18]. Additionally, nivolumab + ipilimumab is also considered to be a preferential choice to treat poor- or intermediate-risk patients with clear cell mRCC. However, cabozantinib monotherapy is also acknowledged as a preferential treatment for poor-/intermediate-risk patients according to this organisation, with a 2A evidence level [18].

Finally, the 2021 update of the European Association of Urology (EAU) guidelines also gives immune-based combinations a central role in the clear cell mRCC treatment, recommending pembrolizumab + axitinib, nivolumab + cabozantinib or pembrolizumab + lenvatinib as the SOC for all patients with clear cell mRCC, and the nivolumab + ipilimumab combination in the poor-/intermediate-risk patients (all with a $1 \mathrm{~b}$ evidence level) [17]. As in the ESMO guidelines, TKI monotherapy is relegated to an alternative option for patients who cannot receive or tolerate ICIs.

\section{Currently Recommended Combination Treatments: Efficacy and Safety Profile}

In this section, we review the main evidence available to date concerning the use of combinations to treat clear cell mRCC. We focus on nivolumab + ipilimumab, pembrolizumab + axitinib, nivolumab + cabozantinib and pembrolizumab + lenvatinib, which are recommended by the three organisations cited earlier [16-18], and are the ones with the greatest amount of accumulated evidence.

The pivotal study that led to the approval and subsequent recommendation of nivolumab + ipilimumab was CheckMate-214 [24-26]. The authors of this phase 3 clinical trial analysed the outcomes and safety profile of 1096 patients with previously untreated clear cell mRCC, who were randomised to receive nivolumab $3 \mathrm{mg} / \mathrm{kg}+$ ipilimumab $\quad 1 \mathrm{mg} / \mathrm{kg}$ every 3 weeks for four doses, followed by nivolumab $3 \mathrm{mg} / \mathrm{kg}$ every 2 weeks, or sunitinib $50 \mathrm{mg}$ once daily in 6-week cycles (4 weeks on, 2 weeks off) [24-26]. The primary endpoints of this trial were OS, ORR and PFS in the IMDCdefined intermediate-/poor-risk patients, which comprised approximately $77 \%$ of the intention to treat (ITT) population. The latest data release corresponds to a median follow-up of 55 months and clearly demonstrates the superiority of nivolumab + ipilimumab over sunitinib in the intermediate-/poor-risk population: in fact, the risk of death for patients in the experimental arm was 35\% lower than that of patients in the control arm (HR 0.65; 95\% CI 0.54-0.78) [26]. Moreover, despite showing a delayed benefit, PFS was also significantly longer in nivolumab + ipilimumab-treated patients, with 4 -year probabilities of $32.7 \%$ vs $12.3 \%$ (HR 0.74; 95\% CI 0.62-0.88). Finally, the ORR was higher among patients in the experimental arm $(41.9 \%$ vs $26.8 \%, p<0.0001)$, as was the proportion of patients achieving a complete response $(10.4 \%$ vs $1.4 \%)$, and the duration of response (HR $0.45 ; 95 \%$ CI 0.31-0.65) [26].

The pembrolizumab + axitinib combination was addressed in the KEYNOTE-426 trial, which included 861 treatment-naïve patients with clear cell mRCC $[30,31]$. These patients were 
randomised to receive pembrolizumab $200 \mathrm{mg}$ once every 3 weeks + axitinib $5 \mathrm{mg}$ twice daily or sunitinib $50 \mathrm{mg}$ once daily in 6-week cycles ( 4 weeks on, 2 weeks off) [30, 31]. OS and PFS were the primary endpoints, and the outcomes of both supported the superiority of the combination treatment compared with sunitinib. With a median follow-up of 30.6 months, the HR for OS was 0.68 (95\% CI $0.55-0.85$; $p=0.0003)$, while the HR for PFS was $0.71(95 \%$ CI $0.60-0.84 ; p<0.0001)$ [31]. The ORR in pembrolizumab + axitinib-treated patients was significantly higher than that in sunitinib-treated patients (60\% vs $40 \%$; $p<0.0001)$, as was the percentage of patients with complete response (9\% vs 3\%) and the median duration of response (23.5 vs 15.9 months) [31].

CheckMate-9ER was the phase 3 clinical trial that addressed the efficacy and toxicity of the nivolumab + cabozantinib combination [32]. Briefly, 651 patients with previously untreated clear cell mRCC were randomised to receive nivolumab $240 \mathrm{mg}$ every 2 weeks + cabozantinib $40 \mathrm{mg}$ once daily or sunitinib $50 \mathrm{mg}$ once daily in 6-week cycles (4 weeks on, 2 weeks off) [32]. After a median follow-up of 18.1 months, the primary endpoint (PFS) was met, with a median PFS of 16.6 months among patients in the experimental arm versus 8.3 months in the control arm (HR 0.51; 95\% CI 0.41-0.64; $p<0.001)$. There was also a significant difference in OS: the risk of death in nivolumab + cabozantinib-treated patients was $40 \%$ lower than that of sunitinib-treated patients (HR 0.60; 98.89\% CI, 0.40-0.89; $p=0.001$ ). Accordingly, the ORR reported for patients in the experimental arm was higher than that of patients in the control arm $(55.7 \%$ vs $27.1 \% ; p<0.001)$, as was the complete response rate $(8.0 \%$ vs $4.6 \%)$ and the median duration of response (20.2 vs 11.5 months) [32].

The pembrolizumab + lenvatinib combination was evaluated in the CLEAR trial of 1069 patients with previously untreated clear cell mRCC [29]. Patients were randomised to receive lenvatinib $20 \mathrm{mg}$ once daily + pembrolizumab $200 \mathrm{mg}$ every 3 weeks, lenvatinib $18 \mathrm{mg}$ once daily + everolimus $5 \mathrm{mg}$ once daily, or sunitinib $50 \mathrm{mg}$ once daily in 6-week cycles (4 weeks on, 2 weeks off) [29]. After a median follow-up of 26.6 months, median PFS (primary endpoint) was longer with pembrolizumab + lenvatinib versus sunitinib (23.9 vs 9.2 months; HR 0.39; 95\% CI $0.32-0.49 ; \quad p<0.001)$. Pembrolizumab + lenvatinib-treated patients had improved OS versus sunitinib-treated patients, with risk of death being 34\% lower with pembrolizumab + lenvatinib (HR 0.66; 95\% CI $0.49-0.88 ; p=0.005)$. Compared with sunitinib, pembrolizumab + lenvatinib was associated with a higher ORR (71.0\% vs $36.1 \%)$, complete response rate $(16.1 \%$ vs $4.2 \%)$ and median duration of response (25.8 vs 14.6 months) [29].

Although treatment combinations brought an undeniable benefit to many patients with clear cell mRCC, they did so at the cost of increased toxicity compared with single-agent therapy. Even though a greater proportion of the sunitinib-treated CheckMate-214 patients suffered grade 3 or 4 treatment-related adverse events (TRAEs) $(64.1 \%$ vs $47.9 \%$ of the patients treated with nivolumab + ipilimumab), the percentage of TRAEs leading to discontinuation was higher in the experimental arm $(22.7 \%$ vs $13.1 \%$ in the control arm) [26]. As for the KEYNOTE-426 trial, the incidence of serious TRAEs was higher among patients treated with the combination ( $28 \%$ vs $16 \%$ ) [31]. Moreover, $21 \%, 20 \%$ and $7 \%$ of patients in the experimental arm discontinued pembrolizumab, axitinib and both drugs, respectively, because of the presence of TRAEs, whereas this percentage was $12 \%$ among sunitinib-treated patients [31]. In CheckMate-9ER, grade 3 or higher TRAEs occurred in $60.6 \%$ of the nivolumab + cabozantinib-treated patients and $50.9 \%$ of the sunitinib-treated patients [32]. Additionally, the percentage of patients who discontinued the treatment because of AEs was $19.7 \%$ in the experimental arm $(6.6 \%$ of patients discontinued nivolumab only, 7.5\% discontinued cabozantinib only and $5.6 \%$ discontinued both) and $16.9 \%$ in the control arm [32]. Lastly, in CLEAR, the incidence of grade 3 or higher TRAEs was $71.6 \%$ with pembrolizumab + lenvatinib and $58.8 \%$ with sunitinib, and AEs led to treatment discontinuation in $37.2 \%$ and $14.4 \%$ of patients, respectively [29]. 
Two meta-analyses were carried out to address the toxicity of the combination treatments from a global perspective. One of them, by Quhal et al., included six phase 3 trials and 5121 patients, and considered the following combinations: nivolumab + ipilimumab, avelumab + axitinib, pembrolizumab + axitinib, atezolizumab + bevacizumab, nivolumab + cabozantinib and pembrolizumab + lenvatinib (Table 1) [36]. When compared with sunitinib-treated patients, those treated with nivolumab + ipilimumab had a significantly higher likelihood of discontinuing treatment because of TRAEs, and of having hyperthyroidism, adrenal insufficiency, pneumonitis, colitis and elevated alanine transaminase (ALT) and aspartate transaminase (AST) levels. Likewise, pembrolizumab + axitinibtreated patients had a significantly higher likelihood of having hyperthyroidism, adrenal insufficiency, pneumonitis, colitis, diarrhoea, hand-foot syndrome and elevated ALT and AST levels. Nivolumab + cabozantinib-treated patients had a significantly higher likelihood of discontinuing treatment because of TRAEs. Similarly, pembrolizumab + lenvatinib-treated patients had a significantly higher likelihood of treatment discontinuation due to TRAEs, and of having hyperthyroidism, adrenal insufficiency, pneumonitis, diarrhoea and elevated AST levels. Of note, when compared with sunitinib, all combinations were associated with significantly lower rates of haematological adverse events (AEs) (namely neutropenia, anaemia and thrombocytopenia) [36].

The second meta-analysis, by Rizzo et al., focused on the incidence of gastrointestinal AEs and considered 3059 patients from four phase 3 trials, treated with either sunitinib or one of the following combinations: pembrolizumab + axitinib, nivolumab + cabozantinib, avelumab + axitinib and pembrolizumab + lenvatinib (Table 1) [37]. When compared with sunitinib-treated patients, those treated with pembrolizumab + axitinib or pembrolizumab + lenvatinib had a significantly higher likelihood of experiencing allgrade diarrhoea, grades 3-4 diarrhoea and grades 3-4 decreased appetite. Moreover, the likelihood of experiencing all-grade diarrhoea was also significantly higher in nivolumab + cabozantinib-treated patients. The likelihood of grades 3-4 nausea and allgrade decreased appetite was higher with pembrolizumab + lenvatinib; however, the likelihood of all-grade nausea was lower with pembrolizumab + axitinib [37].

\section{Risk Stratification and its Clinical Implications: Patients with a Favourable Prognosis}

Except for nivolumab + ipilimumab, the recommendations made by the international guidelines concerning the utilisation of treatment combinations are independent of the patients' prognostic risk. This is because the primary efficacy population of CheckMate-214 was limited to poor-/intermediate-risk patients, while the primary efficacy population of both KEYNOTE-426 and CheckMate-9ER included patients classified in all IMDC risk categories. However, the benefit demonstrated by favourable-risk patients when treated with combination treatments seems to be smaller than that shown by those in the intermediate- or poorrisk categories, sometimes lacking significance in its comparison with sunitinib-treated patients. This trend is consistent across all recommended combinations, as shown by a post hoc pooled analysis [38] and as is briefly outlined below for each study.

In CheckMate-214, 23\% of the patients allocated to each arm were of favourable risk (as defined by the IMDC prognostic model) $[24,25]$. Although excluded from the primary efficacy analysis, these patients were nevertheless included in the ITT population and their outcomes were analysed. Subgroup analyses revealed an almost overlapping pattern of the OS and PFS survival curves of both treatment arms among favourable-risk patients: at 48 months, $65.1 \%$ of patients were alive in the nivolumab + ipilimumab arm versus $68.9 \%$ in the sunitinib arm (HR 0.93; 95\% CI 0.62-1.40), and $25.4 \%$ versus $31.6 \%$, respectively, had not progressed (HR 1.84; 95\% CI 1.29-2.62) [26]. In fact, the OS and PFS outcomes of favourable-risk patients treated with sunitinib were numerically 


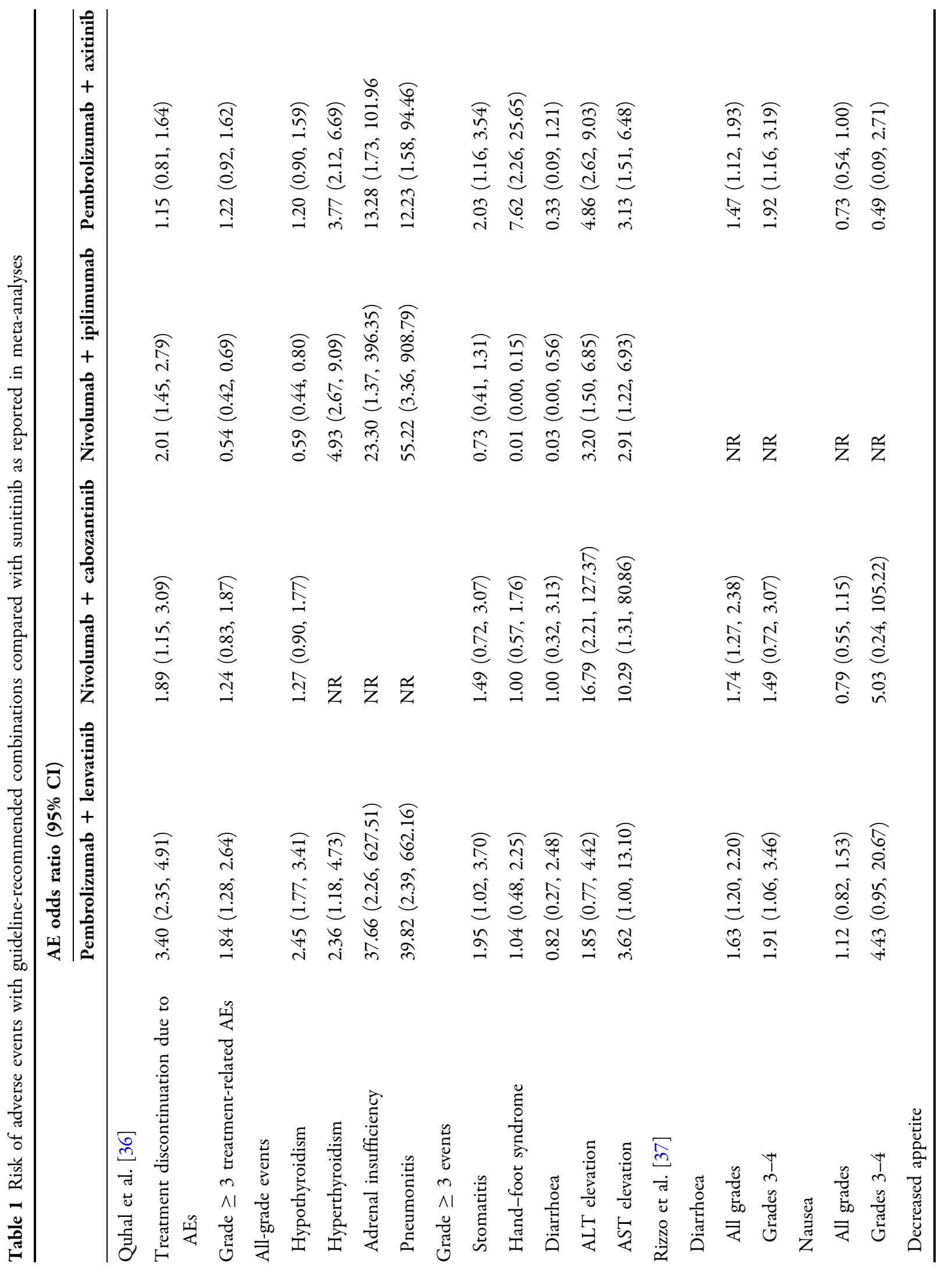


superior to those treated with the nivolumab + ipilimumab combination. Moreover, the ORR at 4 years was significantly higher in sunitinib-treated patients $(51.6 \%$ vs $29.6 \%$ in the experimental arm, $p=0.0005)$, although the rates of complete response and duration of response favoured nivolumab + ipilimumab [26]. Additionally, a post hoc analysis of the CheckMate-214 population stratified by IMDC risk factors showed that the ORR among patients with no risk factors was actually lower with nivolumab + ipilimumab than with sunitinib (39\% vs 50\%) [39]. Furthermore, while the ORR in sunitinib-treated patients decreases progressively as the number of risk factors increases, the ORR with nivolumab + ipilimumab is approximately the same for all patient risk categories, being actually slightly lower for patients in the favourable-risk category (39\% vs $40-44 \%$ for patients with 1-6 risk factors) [39].

The KEYNOTE-426 population included approximately $31 \%$ of patients with a favourable-risk prognosis, as defined by the IMDC criteria [30, 31]. As in CheckMate-214, the OS curves of these patients have an overlapping pattern: at 2 years, $85.3 \%$ of the patients treated with the combination were still alive, which was slightly lower than the $87.7 \%$ observed among sunitinib-treated patients (HR 1.06; 95\% CI $0.60-1.86 ; p=0.58$ ) [31]. In the prespecified subgroup analysis by IMDC risk category, the OS benefit was only evident in the intermediate-/poor-risk group (HR 0.63; 95\% CI $0.50-0.81 ; p<0.001)$ [31]. In the PFS analysis by IMDC risk category, PFS benefits with pembrolizumab + axitinib were generally consistent across the risk categories, although the results in the favourable-risk category did not reach statistical significance (HR 0.79; 95\% CI $0.57-1.09 ; p=0.078$ ). However, a post hoc subgroup analysis showed that the ORR benefit with pembrolizumab + axitinib was consistent across all IMDC risk categories [31].

Approximately $22 \%$ of the 651 patients included in the CheckMate-9ER had an IMDCdefined favourable prognosis [32]. In the prespecified subgroup analysis by IMDC risk category, patients in the favourable-risk category had a borderline significant PFS benefit with 
nivolumab + cabozantinib (HR 0.62; 95\% CI 0.38-1.01), although the associated HR was higher than that of the intermediate-risk (HR 0.54 ; 95\% CI 0.40-0.72) and poor-risk (HR 0.37; 95\% CI 0.23-0.58) categories. For OS, there was no significant benefit with nivolumab + cabozantinib in the favourablerisk (HR 0.84; 95\% CI 0.35-1.97) or intermediate-risk (HR 0.70; 95\% CI 0.46-1.07) categories, but a significant OS benefit was observed among patients in the poor-risk category (HR 0.37; 95\% CI 0.21-0.66). Of note, and as reported in KEYNOTE-426, the likelihood of having a higher ORR with nivolumab + cabozantinib versus sunitinib was observed consistently in patients in the favourable-risk (OR 25.9; 95\% CI 9.8-40.2), intermediate-risk (OR 28.2; 95\% CI 18.3-37.3) and poor-risk (OR 30.5; 95\% CI 16.0-43.9) categories [32].

In the CLEAR study, approximately 33\% of patients were in the favourable IMDC prognostic risk group [29]. In the prespecified subgroup analysis by IMDC risk category, the PFS benefit with pembrolizumab + lenvatinib was observed across all risk categories, although the HR was slightly higher in the favourable-risk subgroup (HR 0.41 ; 95\% CI 0.28-0.62) than in the intermediate-risk (HR 0.39; 95\% CI 0.29-0.52) or poor-risk (HR 0.28; 95\% CI 0.13-0.60) subgroups. Similar to the CheckMate-9ER, significant OS benefit was observed in the poor-risk subgroup (HR 0.30; 95\% CI 0.14-0.64), but not in the favourable-risk (HR 1.15; 95\% CI $0.55-2.40$ ) or intermediate-risk (HR $0.72 ; 95 \%$ CI 0.50-1.05) subgroups [29].

\section{Non-Clear Cell RCC}

The medical management of patients with nonclear cell RCC remains a particularly challenging issue. As these subtypes are seldom included in phase 3 trials, the treatment strategies employed are often an extrapolation of what has been evaluated and approved in the setting of the clear cell histology. However, the outcomes of patients with non-clear cell RCC treated with the currently approved systemic therapies are limited, and usually are significantly inferior to those of patients with clear cell RCC. Acknowledging this fact, both the National Comprehensive Cancer Network (NCCN) and the EAU guidelines recommend inclusion of patients with non-clear cell RCC in clinical trials whenever appropriate $[17,18]$. However, both of these organisations also recommend the use of sunitinib, based on data from three phase 2 trials that reported a tendency for superiority of this anti-VEGF TKI compared with everolimus (the ESPN [40], ASPEN [41] and RECORD-3 [42] trials). The NCCN guidelines also recommend cabozantinib [18].

The ESMO guidelines are more detailed regarding the management of the non-clear cell RCC subtype [43]. While they maintain the recommendation of including these patients in appropriate clinical trials, cabozantinib is the preferred first-line treatment option for patients with papillary mRCC without additional molecular testing (level II, B) [43]. The SWOG PAPMET trial demonstrated a PFS benefit for cabozantinib over sunitinib (9.0 vs 5.6 months; HR 0.60; 95\% CI 0.37-0.97; $p=0.02$ ) and higher ORR (23\% vs 4\%) [44]. Alternative options include sunitinib (level II, B) and pembrolizumab (level III, B; based on KeyNote-426 $[30,31])$ without further molecular testing, and savolitinib in MET-driven tumours, where available (level III, C; based on SAVOIR trial [45]) [43]. In fact, in the era of precision oncology, results point to a SOC in non-clear cell RCC that will, in future, be tailored to the specific histology within this broad group of tumours. For example, in patients with papillary type RCC, as stated above, the SWOG PAPMET Trial suggested a PFS benefit with cabozantinib over sunitinib [44], while savolitinib showed promising efficacy compared with sunitinib in the SAVOIR study [45], crizotinib showed sustained disease control in CREATE [46] and foretinib demonstrated antitumour activity in a phase 2 study [47]. These data suggest that treatment preferences for this specific subtype of tumour may change in the future.

Concerning combination therapy in patients with non-clear cell RCC, data are still scarce. The only trial specifically focused on non-clear cell RCC was reported by Gupta et al. [48]. In 
this study, nivolumab + ipilimumab was used to treat a small population of 18 patients with varying histological subtypes (papillary, chromophobe, unclassified, renal adenocarcinoma, translocation and medullary). The results were positive overall, with an ORR of $33.3 \%$, a median duration of response of 4.3 months, a median PFS of 7.1 months and a 12-month OS of $64.2 \%$ [48]. However, these values are numerically similar to those obtained using anti-VEGF TKIs. Moreover, the efficacy of this combination may vary with the specific subtype of non-clear cell RCC. Tachibana et al. recently highlighted this aspect by showing that the effect of nivolumab + ipilimumab in papillary RCC was inferior to that demonstrated in patients with clear cell RCC, with a lower ORR $(14.2 \%$ vs $52.1 \%, p=0.06)$ and a shorter median PFS (2.4 vs 28.1 months, $p=0.014$ ) [49]. Additionally, Tykodi et al. recently presented the outcomes with nivolumab + ipilimumab treatment in a population of patients with previously untreated non-clear cell mRCC [50]. Although based on a small sample $(n=52)$, these results were rather promising, showing a median PFS of 3.7 months and a median OS of 21.2 months [50].

For all the other combinations, the scarcity of data prevents recommendations in the nonclear cell RCC setting. Therefore, as it currently stands, considering an approach with antiVEGF TKI monotherapy may provide non-inferior benefits with less toxicity than an ICI-based combination.

\section{EMERGING BIOMARKERS}

As the number of available systemic therapies increases, therapeutic decision-making has become more complex. Although the MSKCC and IMDC models have value as prognostic markers, their predictive ability regarding treatment sensitivity is suboptimal. Therefore, identifying surrogate markers to categorise tumours according to their main genetic features and molecular pathways, aiding in the selection of the most suitable targeted agents, has long been the focus of several researchers in the RCC field. The many biomarker candidates being explored have been extensively reviewed elsewhere [51-53] and will not be addressed here. However, a brief comment should be made concerning PD-L1. Given its role in the immune checkpoint blockade, PD-L1 is probably one of the most studied potential biomarkers for PD-1/PD-L1-targeted therapy responsiveness. Despite being acknowledged as a negative prognostic factor, its ability to predict outcomes with ICI therapy is still debatable. In fact, the data gathered to date are limited by the utilisation of different assessment methods and by tumour heterogeneity, making them insufficient to validate PD-L1 as a predictive biomarker [51, 53].

Omics-based approaches are now emerging in the RCC setting in an attempt to identify biologically driven patient subgroups. Considering the role of angiogenesis and immune blockade in RCC tumour development, as well as the complex crosstalk between these two processes, the utilisation of genomics or transcriptomics seems like a sensible strategy to capture the multifaceted nature of this disease. Two recent studies have employed such techniques in the context of combination treatments and will be briefly reviewed here.

One of those studies was the phase 2, openlabel BIONIKK trial, which compared the efficacy and safety of nivolumab versus nivolumab + ipilimumab versus TKI monotherapy in patients with treatment-naïve clear cell mRCC [54]. In BIONIKK, 202 patients were categorised according to the expression of a previously defined 35-gene signature; the resulting groups (ccRcc1-4) were not correlated with IMDC risk categories. Patients belonging to ccRcc1 (immune-low) and ccRcc4 (immunehigh) were randomised to receive nivolumab or nivolumab + ipilimumab, whereas patients belonging to ccRcc2 (angio-high) and ccRcc3 (normal-like) were randomised to receive nivolumab + ipilimumab or a TKI. The ORR (primary endpoint) was comparable across all subgroups for patients receiving nivolumab + ipilimumab, reported as 39\%, $48 \%, 25 \%$ and $53 \%$ in the ccRcc1, ccRcc 2 , ccRcc3 and ccRcc4 groups, respectively. Interestingly, in the ccRcc1 group, the ORR with nivolumab monotherapy was almost half of 
that with nivolumab + ipilimumab (21\% vs $39 \%)$, whereas these values were comparable in the ccRcc4 group (50\% vs 53\%). Moreover, TKItreated patients had an ORR similar to that of nivolumab + ipilimumab-treated patients in the ccRcc 2 group ( $54 \%$ vs $48 \%$ ), but the ORR was clearly lower in the ccRcc3 group (0\% vs 25\%) [54].

Another study, IMmotion 150, included 305 patients with previously untreated mRCC who were randomised to receive atezolizumab monotherapy or atezolizumab + bevacizumab vs sunitinib [55]. This prospective trial included an exploratory biomarker analysis based on the differential expression of a previously defined group of genes related to angiogenesis (Angio $^{\text {Low }} /$ Angio $\left.^{\text {High }}\right)$, T-effector and interferongamma activity $\left(\right.$ Teff $^{\text {Low }} /$ Teff $^{\text {High }}$ ) and myeloid inflammatory response (Myeloid ${ }^{\text {Low }} /$ Myeloid $\left.{ }^{\mathrm{High}}\right)$. Sunitinib outcomes were better in the Angio $^{\text {High }}$ compared with Angio $^{\text {Low }}$ patients, both in terms of ORR (46\% vs $9 \%$ ) and PFS (HR $0.31 ; 95 \%$ CI 0.18-0.55). Furthermore, in Angio $^{\text {Low }}$ patients, PFS was longer with atezolizumab + bevacizumab than with sunitinib (HR 0.59; 95\% CI 0.35-0.98). Conversely, TeffHigh patients treated with atezolizumab + bevacizumab had an improved ORR (49\% vs $16 \%)$ and PFS (HR 0.50; 95\% CI 0.30-0.86) when compared with Teff ${ }^{\text {Low }}$ patients treated with the same combination. Accordingly, PFS outcomes in atezolizumab + bevacizumab-treated patients were better than those of sunitinibtreated patients in the $\mathrm{Teff}^{\mathrm{High}}$ population (HR 0.55; 95\% CI 0.32-0.95). Finally, Myeloid ${ }^{\text {High }}$ patients had a worse PFS outcome compared with Myeloid ${ }^{\text {Low }}$ patients when treated with atezolizumab (HR 2.98; 95\% CI 1.68-5.29]) or atezolizumab + bevacizumab (HR 1.71; 95\% CI 1.01-2.88), and atezolizumab-treated Myeloid $^{\text {High }}$ patients had a shorter PFS than sunitinib-treated Myeloid ${ }^{\text {High }}$ patients (HR 2.03; 95\% CI 1.21-3.40) [55].

While both the aforementioned outlined studies had several limitations and require further validation, they suggest that gene expression signatures may hold some discriminatory potential in terms of responsiveness to antiangiogenic and ICI treatments. Therefore, molecular-based selection of patients may, in the long run, be the key to personalised medicine in this challenging context.

\section{PATIENTS' PREFERENCES AND OTHER FACTORS WITH IMPACT ON THE THERAPEUTIC DECISION PROCESS}

The plethora of agents and combinations currently available to treat patients with mRCC makes the therapeutic decision-making process in this setting particularly complex. Along with efficacy and tolerability, patients' health-related QoL and preferences should also be taken into account. Whereas the former is intimately related to tumour burden, disease progression and treatment safety, the latter is based on the individual perceptions of the risks and benefits of treatment. The studies conducted on this issue have highlighted that patients usually attribute a considerable weight to treatment efficacy, even at the expense of moderate toxicity and administration comfort. In an online survey carried out in September 2020 that included 1136 patients with kidney cancer (411 of whom were on systemic therapy), complete response was chosen as the most important outcome for treatment selection by $58.8 \%$ of the respondents [56]. Of note, only $5.7 \%$ and $3.7 \%$ of all patients chose low risk of toxicity and chance of discontinuing therapy, respectively. Moreover, $42.1 \%$ of patients preferred oral therapy versus $10.9 \%$ who preferred infusion therapy; however, nearly half of the respondents $(47 \%)$ were indifferent to the route of administration. Additionally, $86.3 \%$ and $71.7 \%$ of all patients defined treatment success as tumour size reduction and stable disease, respectively; better QoL and symptom control were part of this definition for $47.7 \%$ and $35.1 \%$ of the respondents, respectively [56]. Similarly, PFS was ranked as the most important feature in a survey of 138 patients with RCC [57], whereas the probability of living for 3 years or more was considered to be the most important outcome of RCC treatment in a questionnaire completed by 201 patients and 142 physicians [58]. 
Interestingly, Mansfield et al. demonstrated that the level of patient information was correlated with their willingness to accept treatment-related toxicities [59]. In fact, their survey of 378 patients with RCC (of whom 50\% had advanced disease and $31 \%$ were on systemic therapy) showed that well-informed patients were more prone to accept treatment-related toxicities, and preferred treatments with a higher chance of having a longer PFS even at the expense of tolerability [59].

\section{mRCC Management in the Context of COVID-19 (Coronavirus Disease 2019) Pandemic}

By November 2021, the ongoing severe acute respiratory syndrome coronavirus 2 (SARS-CoV2) pandemic had been responsible for over 252 million cases and over 5 million deaths globally [60]. This major public health emergency represents a unique challenge for oncologists for several reasons. Patients with cancer are more likely to be infected by SARS-CoV-2 and to develop severe infections and die as a result of COVID-19. Moreover, the delivery of cancer care has been severely impacted by the limitation and prioritisation of healthcare resources [61, 62]. In this context, physicians have been asked to reassess cancer treatment risks and benefits, while weighing the risks of COVID-19 infection. This reassessment is particularly relevant for immunotherapy-based approaches, given the unique immune disruptive effect attributed to SARS-CoV-2. So far, based on the available evidence, the risk of COVID-19-associated immune dysregulation in patients with solid tumours seems to be similar to that of the general population [63]. Moreover, ICIs do not seem to predispose patients with COVID-19 and cancer to worse outcomes [64]. However, further studies are needed to understand the risk-benefit relationship of immunotherapy during the ongoing SARS-CoV2 pandemic.

Not surprisingly, many physicians and oncology care centres adapted their treatment algorithms to consider the added risk of COVID19 infection. In the specific case of RCC, a survey of 41 experts revealed a decrease in the use of combination therapy, favouring the prescription of TKI monotherapy [65]. Indeed, while pembrolizumab + axitinib was the preferred treatment option in favourable-risk patients for $53 \%$ of respondents before the pandemic, only $38 \%$ maintained this preference during the pandemic. Conversely, only $13 \%$ of the experts preferred single-agent sunitinib or pazopanib to treat these patients before the pandemic, increasing to $35 \%$ during the pandemic $(p<0.001)$. As for fit and intermediate-/ poor-risk patients, nivolumab + ipilimumab and pembrolizumab + axitinib were the preferred treatment options in the opinion of $80 \%$ and $18 \%$ of respondents, respectively, before the pandemic. Amidst the COVID-19 risk, the proportion who preferred the ICI/ICI combination decreased to $41 \%$, whereas the proportion who preferred the ICI/VEGF TKI combination increased to $30 \%$, and $29 \%$ reported a preference for TKI monotherapy (sunitinib, pazopanib, tivozanib or cabozantinib) [65].

In the context of the pandemic, the NCCN has issued a number of suggestions focused on the management of COVID-19 in patients with cancer [66]. These experts highlight the lack of robust data concerning ICI therapies. While suggesting that interruption of ICI therapy may be advisable in patients with cancer who develop COVID-19, they also consider that this decision should be individualised and should consider the specific ICI and the COVID-19 severity [66]. Additionally, ESMO published a consensus statement aimed at guiding cancer care amidst the COVID-19 pandemic [67]. Among their many recommendations, these experts advocated that ICI treatment should be interrupted when a patient tests positive for SARS-CoV-2 and resumed upon complete resolution of the infection [67]. Moreover, TKI therapy can be withheld in patients with a low tumour burden in the presence of a severe COVID-19 infection and when their oncological disease is stable. Furthermore, in a specific set of recommendations issued exclusively for the management of patients with RCC, ESMO experts recommend that ICI/VEGF TKI or ICI/ ICI combinations should be maintained as the SOC for patients with IMDC intermediate- or 
poor-risk prognosis, an exception made to particularly challenging healthcare environments [68]. Conversely, VEGF-targeted oral therapy is considered to be an appropriate choice for patients with favourable-risk prognosis when the risk of ICIs is considered to be too high during acute phases of the pandemic [68].

\section{EXPERT COMMENT}

The advent of ICIs and their utilisation in combination with VEGF-targeted therapy have revolutionised the treatment landscape of patients with mRCC. The benefits in terms of survival and response rates are undeniable and have brought renewed hope for the management of this disease. However, as often occurs in oncology care, one size does not fit all. In the absence of predictive biomarkers in clinical practice, international guidelines support basing the therapeutic decision on the patient's clinical risk. Yet, the tools used to evaluate this risk were developed when the SOC was either a non-specific immune approach (MSKCC) or VEGF-targeted single agents (IMDC). Although these models seem to maintain their prognostic ability, they are less accurate when it comes to differentiating responsiveness to new combination strategies. Therefore, in the era of personalised healthcare, physicians should attempt to frame the major clinical trial results alongside patient comorbidities, concomitant medications, willingness and ability to withstand treatment AEs, preferences in terms of valued outcomes and route of administration, drug accessibility and costs. An ideal therapy should attain maximum efficacy, while avoiding overtreatment and unnecessary toxicity. On the basis of this premise and on the evidence reviewed above, we strongly believe that TKI monotherapy still holds a key role in mRCC management and should be considered as the preferential frontline alternative to treat the following patients:

- Favourable-risk patients with a low tumour burden and with an indolent disease progression pattern: In these patients, who have a good prognosis and who are not in an immediate need of response, both the ICI/ICI and ICI/ VEGF TKI combination options may represent overtreatment, the benefits of which do not justify the associated increased toxicity risk. In fact, as seen in the subgroup analyses outlined earlier, the PFS and OS benefits in favourable-risk patients treated with these combinations are consistently inferior or similar to those of the unselected population. In the case of ICI/VEGF TKI therapy, the utilisation of two mechanisms of action may elicit resistance to both treatments, thereby limiting the sequential options for subsequent therapy lines, a setting in which the lack of evidence is particularly worrisome. Of note, this recommendation for the use of TKI monotherapy excludes patients who, despite having favourable risk, have a high tumour burden or are extremely symptomatic. These patients might benefit from the higher ORRs observed with combination treatment. In selected patients with indolent low-volume disease, active surveillance may also be an option.

- Patients with a clearly defined angiogenic profile: While angiogenesis and immune blockade are two hallmarks of RCC, studies on potential biomarkers and molecular profiles suggest that their relative relevance varies between patients. Treatment outcomes in patients with mostly angiogenesis-driven tumours are expected to be maximised when a single anti-VEGF agent is used. While the current lack of available data hampers the identification of these patients during routine clinical practice, future developments in this field should allow for the differentiation of angiogenic from immunogenic tumours, thereby guiding the therapeutic decisionmaking process from a biological perspective.

- Patients who have non-clear cell RCC: Given the lack of data concerning this rare subset of patients, we believe that patients with nonclear cell RCC should either be enrolled in clinical trials or be treated with standard TKI monotherapy. As previously advocated for patients with a favourable-risk prognosis, the utilisation of a single mechanism may have the advantage of saving alternative 
treatments for later therapy lines, which could otherwise be exhausted by the development of resistance mechanisms.

- Patients who are ineligible for combination treatment, either because of the presence of significant comorbidities (namely, immunosuppressed transplant recipients or those with severe autoimmune diseases) or potential drug interactions (e.g. high-dosage steroids), or in patients who are unable to tolerate the added toxicity associated with dual-agent treatment (e.g. elderly and unfit patients): A single anti-VEGF drug is likely to be a sensible choice for these patients, given its enhanced tolerability when compared with combination regimens.

- Well-informed and educated patients who prefer to be treated with a VEGF TKI: VEGF TKIs are orally administered drugs with a generally manageable safety profile that seldom require hospitalisation; this option may be preferred by patients who value being at home and would rather be on oral therapy only (compared with intravenous therapy). Also, as these drugs can be managed through a telehealth approach or from an outpatient clinic, this option may be particularly desirable in the context of limited healthcare resources during the current SARS-CoV-2 pandemic.

\section{ACKNOWLEDGEMENTS}

Funding. Mário Fontes-Sousa, Alicia Oliveira, Filipa Palma dos Reis, Pedro Silvestre Madeira and Sara Meireles were paid consultants to Pfizer in connection with the development of this manuscript. The Journal's Rapid Service and Open Access Fees were funded by Pfizer.

Medical Writing, Editorial and Other Assistance. Medical Writing and Editorial Assistance in the preparation of this article was provided by Catarina L. Santos, PhD, on behalf of Springer Healthcare, Iberica. Sarah Greig, $\mathrm{PhD}$, of Springer Healthcare Communications, provided editorial assistance with post-submission revisions. Support for this assistance was funded by Pfizer. The authors would like to acknowledge all the advisors present in the meetings that originated this article, whose opinions and shared expertise were a great contribution to the discussion: Alina Rosinha, Ana Faria, Ângela Novais Dias, Bruno Figueiredo; Cidália Pinto, Gonçalo Atalaia, Joana Febra, João Moreira Pinto, Juan Mellidez, Lorenzo Marconi, Margarida Miguens, Mariana Malheiro, Mónica Mariano, Sandra Custódio, Sílvia Lopes and Sofia Oliveira. Moreover, the authors are deeply indebted to Thomas Powles for his helpful insights in the topic under discussion.

Authorship. All named authors meet the International Committee of Medical Journal Editors (ICMJE) criteria for authorship for this article, take responsibility for the integrity of the work as a whole, and have given their approval for this version to be published.

Author Contributions. Mário Fontes-Sousa was responsible for the study conception and design. All authors contributed to literature search, data analysis and critical revisions of the manuscript. All authors read and approved the final version for publication.

Disclosures. Mário Fontes-Sousa has been contracted as a consultant/speaker for Astellas, Bristol Myers Squibb, Merck Sharp \& Dohme (MSD), Novartis, Pfizer and Roche. Filipa Carneiro has received honoraria/consultation fees from Jansen, Astellas, Bristol Myers Squibb, Bayer, Ipsen, MSD and Pfizer. Helena Magalhães has received advisory board fees from Pfizer and Janssen. PSM has provided consulting or advisory services to Bristol Myers Squibb, Ipsen, MSD and Pfizer; has received research funding from Bristol Myers Squibb, MSD and Pfizer; has received honoraria from Bristol Myers Squibb, Ipsen and MSD; and has received travel, accommodations or expenses from Bristol Myers Squibb, Ipsen, MSD, Novartis and Pfizer. Sara Meireles has provided consulting or advisory services to Bayer, Incyte, Ipsen, Janssen, 
Merck Sharp \& Dohme (MSD), Novartis, Pfizer, Roche and Sanofi.

Compliance with Ethics Guidelines. This article is based on previously conducted studies and does not contain any new studies with human participants or animals performed by any of the authors.

Data Availability. Data sharing is not applicable to this article as no datasets were generated or analysed during the current study.

Open Access. This article is licensed under a Creative Commons Attribution-NonCommercial 4.0 International License, which permits any non-commercial use, sharing, adaptation, distribution and reproduction in any medium or format, as long as you give appropriate credit to the original author(s) and the source, provide a link to the Creative Commons licence, and indicate if changes were made. The images or other third party material in this article are included in the article's Creative Commons licence, unless indicated otherwise in a credit line to the material. If material is not included in the article's Creative Commons licence and your intended use is not permitted by statutory regulation or exceeds the permitted use, you will need to obtain permission directly from the copyright holder. To view a copy of this licence, visit http://creativecommons.org/licenses/bync/4.0/.

\section{REFERENCES}

1. Padala SA, Barsouk A, Thandra KC, et al. Epidemiology of renal cell carcinoma. World J Oncol. 2020;11(3):79-87.

2. Capitanio U, Bensalah K, Bex A, et al. Epidemiology of renal cell carcinoma. Eur Urol. 2019;75(1):74-84.

3. Barata PC, Rini BI. Treatment of renal cell carcinoma: current status and future directions. CA Cancer J Clin. 2017;67(6):507-24.

4. Linehan WM, Ricketts CJ. The Cancer Genome Atlas of renal cell carcinoma: findings and clinical implications. Nat Rev Urol. 2019;16(9):539-52.
5. Choueiri TK, Motzer RJ. Systemic therapy for metastatic renal-cell carcinoma. $\mathrm{N}$ Engl J Med. 2017;376(4):354-66.

6. Jonasch E, Gao J, Rathmell WK. Renal cell carcinoma. BMJ. 2014;349:g4797.

7. Zoumpourlis P, Genovese G, Tannir NM, Msaouel P. Systemic therapies for the management of nonclear cell renal cell carcinoma: what works, what doesn't, and what the future holds. Clin Genitourin Cancer. 2021;19(2):103-16.

8. Akhtar M, Al-Bozom IA, Al HT. Papillary renal cell carcinoma (PRCC): an update. Adv Anat Pathol. 2019;26(2):124-32.

9. Lebacle C, Pooli A, Bessede T, Irani J, Pantuck AJ, Drakaki A. Epidemiology, biology and treatment of sarcomatoid RCC: current state of the art. World J Urol. 2019;37(1):115-23.

10. Mouallem NE, Smith SC, Paul AK. Sarcomatoid renal cell carcinoma: biology and treatment advances. Urol Oncol. 2018;36(6):265-71.

11. Motzer RJ, Bacik J, Murphy BA, Russo P, Mazumdar $\mathrm{M}$. Interferon-alfa as a comparative treatment for clinical trials of new therapies against advanced renal cell carcinoma. J Clin Oncol. 2002;20(1): 289-96.

12. Heng DY, Xie W, Regan MM, et al. Prognostic factors for overall survival in patients with metastatic renal cell carcinoma treated with vascular endothelial growth factor-targeted agents: results from a large, multicenter study. J Clin Oncol. 2009;27(34):5794-9.

13. Motzer RJ, Bacik J, Schwartz LH, et al. Prognostic factors for survival in previously treated patients with metastatic renal cell carcinoma. J Clin Oncol. 2004;22(3):454-63.

14. Ko JJ, Xie W, Kroeger N, et al. The International Metastatic Renal Cell Carcinoma Database Consortium model as a prognostic tool in patients with metastatic renal cell carcinoma previously treated with first-line targeted therapy: a population-based study. Lancet Oncol. 2015;16(3):293-300.

15. Massari F, Di Nunno V, Guida A, et al. Addition of primary metastatic site on bone, brain, and liver to IMDC criteria in patients with metastatic renal cell carcinoma: a validation study. Clin Genitourin Cancer. 2021;19(1):32-40.

16. Powles T, Albiges L, Bex A, et al. ESMO Clinical Practice Guideline update on the use of immunotherapy in early stage and advanced renal cell carcinoma. Ann Oncol. 2021. https://doi.org/ 10.1016/j.annonc.2021.09.014. 
17. Ljungberg B, Albiges L, Bedke J, et al. European Association of Urology guidelines on renal cell carcinoma, 2021 update. 2021. https://uroweb.org/ guideline/renal-cell-carcinoma/. Accessed Sep 2021.

18. National Comprehensive Cancer Network. NCCN clinical practice guidelines in oncology (NCCN guidelines)-kidney cancer-version 1.2022-July 1, 2021. 2021. https://www.nccn.org/guidelines/ guidelines-detail?category=1\&id=1440. Accessed Sep 2021.

19. Bedke J, Stuhler V, Stenzl A, Brehmer B. Immunotherapy for kidney cancer: status quo and the future. Curr Opin Urol. 2018;28(1):8-14.

20. Chau V, Bilusic M. Pembrolizumab in combination with axitinib as first-line treatment for patients with renal cell carcinoma (RCC): evidence to date. Cancer Manag Res. 2020;12:7321-30.

21. Daste A, Grellety T, Gross-Goupil M, Ravaud A. Protein kinase inhibitors in renal cell carcinoma. Expert Opin Pharmacother. 2014;15(3):337-51.

22. Escudier B, Albiges L, Sonpavde G. Optimal management of metastatic renal cell carcinoma: current status. Drugs. 2013;73(5):427-38.

23. Motzer RJ, Escudier B, McDermott DF, et al. Nivolumab versus everolimus in advanced renal-cell carcinoma. N Engl J Med. 2015;373(19):1803-13.

24. Motzer RJ, Rini BI, McDermott DF, et al. Nivolumab plus ipilimumab versus sunitinib in first-line treatment for advanced renal cell carcinoma: extended follow-up of efficacy and safety results from a randomised, controlled, phase 3 trial. Lancet Oncol. 2019;20(10):1370-85.

25. Motzer RJ, Tannir NM, McDermott DF, et al. Nivolumab plus ipilimumab versus sunitinib in advanced renal-cell carcinoma. $\mathrm{N}$ Engl J Med. 2018;378(14):1277-90.

26. Albiges L, Tannir NM, Burotto M, et al. Nivolumab plus ipilimumab versus sunitinib for first-line treatment of advanced renal cell carcinoma: extended 4-year follow-up of the phase III CheckMate 214 trial. ESMO Open. 2020;5(6):e001079.

27. Motzer RJ, Robbins PB, Powles T, et al. Avelumab plus axitinib versus sunitinib in advanced renal cell carcinoma: biomarker analysis of the phase 3 JAVELIN Renal 101 trial. Nat Med. 2020;26(11): 1733-41.

28. Rini BI, Powles T, Atkins MB, et al. Atezolizumab plus bevacizumab versus sunitinib in patients with previously untreated metastatic renal cell carcinoma (IMmotion151): a multicentre, open-label, phase 3, randomised controlled trial. Lancet. 2019;393(10189):2404-15.

29. Motzer R, Alekseev B, Rha SY, et al. Lenvatinib plus pembrolizumab or everolimus for advanced renal cell carcinoma. N Engl J Med. 2021;384(14): 1289-300.

30. Rini BI, Plimack ER, Stus V, et al. Pembrolizumab plus axitinib versus sunitinib for advanced renalcell carcinoma. N Engl J Med. 2019;380(12): 1116-27.

31. Powles T, Plimack ER, Soulieres D, et al. Pembrolizumab plus axitinib versus sunitinib monotherapy as first-line treatment of advanced renal cell carcinoma (KEYNOTE-426): extended follow-up from a randomised, open-label, phase 3 trial. Lancet Oncol. 2020;21(12):1563-73.

32. Choueiri TK, Powles T, Burotto M, et al. Nivolumab plus cabozantinib versus sunitinib for advanced renal-cell carcinoma. N Engl J Med. 2021;384(9): 829-41.

33. Roberto M, Botticelli A, Panebianco $M$, et al. Metastatic renal cell carcinoma management: from molecular mechanism to clinical practice. Front Oncol. 2021;11:657639.

34. Mennitto A, Huber V, Ratta R, et al. Angiogenesis and immunity in renal carcinoma: can we turn an unhappy relationship into a happy marriage? J Clin Med. 2020;9(4):930.

35. Motzer RJ, Hutson TE, Cella D, et al. Pazopanib versus sunitinib in metastatic renal-cell carcinoma. N Engl J Med. 2013;369(8):722-31.

36. Quhal F, Mori K, Remzi M, Fajkovic H, Shariat SF, Schmidinger $M$. Adverse events of systemic immune-based combination therapies in the firstline treatment of patients with metastatic renal cell carcinoma: systematic review and network metaanalysis. Curr Opin Urol. 2021;31(4):332-9.

37. Rizzo A, Mollica V, Santoni M, Massari F. Risk of selected gastrointestinal toxicities in metastatic renal cell carcinoma patients treated with immunoTKI combinations: a meta-analysis. Expert Rev Gastroenterol Hepatol. 2021;15(10):1225-32.

38. Lee D, Gittleman H, Weinstock C, et al. An FDApooled analysis of frontline combination treatment benefits by risk groups in metastatic renal cell carcinoma (mRCC) [abstract]. J Clin Oncol. 2021;39(15_suppl):4559.

39. Escudier B, Motzer RJ, Tannir NM, et al. Efficacy of nivolumab plus ipilimumab according to number of IMDC risk factors in CheckMate 214. Eur Urol. 2020;77(4):449-53. 
40. Tannir NM, Jonasch E, Albiges L, et al. Everolimus versus sunitinib prospective evaluation in metastatic non-clear cell renal cell carcinoma (ESPN): a randomized multicenter phase 2 trial. Eur Urol. 2016;69(5):866-74.

41. Armstrong AJ, Halabi S, Eisen T, et al. Everolimus versus sunitinib for patients with metastatic nonclear cell renal cell carcinoma (ASPEN): a multicentre, open-label, randomised phase 2 trial. Lancet Oncol. 2016;17(3):378-88.

42. Motzer RJ, Barrios CH, Kim TM, et al. Phase II randomized trial comparing sequential first-line everolimus and second-line sunitinib versus first-line sunitinib and second-line everolimus in patients with metastatic renal cell carcinoma. J Clin Oncol. 2014;32(25):2765-72.

43. Escudier B, Porta C, Schmidinger M, et al. Renal cell carcinoma: ESMO Clinical Practice Guidelines for diagnosis, treatment and follow-up. Ann Oncol. 2019;30(5):706-20.

44. Pal SK, Tangen C, Thompson IM Jr, et al. A comparison of sunitinib with cabozantinib, crizotinib, and savolitinib for treatment of advanced papillary renal cell carcinoma: a randomised, open-label, phase 2 trial. Lancet. 2021;397(10275):695-703.

45. Choueiri TK, Heng DYC, Lee JL, et al. Efficacy of savolitinib vs sunitinib in patients with MET-driven papillary renal cell carcinoma: the SAVOIR phase 3 randomized clinical trial. JAMA Oncol. 2020;6(8): 1247-55.

46. Schöffski P, Wozniak A, Escudier B, et al. Crizotinib achieves long-lasting disease control in advanced papillary renal-cell carcinoma type 1 patients with MET mutations or amplification. EORTC 90101 CREATE trial. Eur J Cancer. 2017;87:147-63.

47. Choueiri TK, Vaishampayan U, Rosenberg JE, et al. Phase II and biomarker study of the dual MET/ VEGFR2 inhibitor foretinib in patients with papillary renal cell carcinoma. J Clin Oncol. 2013;31(2): 181-6.

48. Gupta R, Ornstein MC, Li H, et al. Clinical activity of ipilimumab plus nivolumab in patients with metastatic non-clear cell renal cell carcinoma. Clin Genitourin Cancer. 2020;18(6):429-35.

49. Tachibana $\mathrm{H}$, Kondo $\mathrm{T}$, Ishihara $\mathrm{H}$, et al. Modest efficacy of nivolumab plus ipilimumab in patients with papillary renal cell carcinoma. Jpn J Clin Oncol. 2021;51(4):646-53.

50. Tykodi SS, Gordan LN, Alter RS, et al. Nivolumab plus ipilimumab in patients with advanced nonclear cell renal cell carcinoma (nccRCC): safety and efficacy from CheckMate 920 [abstract]. J Clin Oncol. 2021;39(6_suppl):309.

51. Argentiero A, Solimando AG, Krebs M, et al. Antiangiogenesis and immunotherapy: novel paradigms to envision tailored approaches in renal cellcarcinoma. J Clin Med. 2020;9(5):1594.

52. D'Aniello C, Berretta $M$, Cavaliere $C$, et al. Biomarkers of prognosis and efficacy of anti-angiogenic therapy in metastatic clear cell renal cancer. Front Oncol. 2019;9:1400.

53. Raimondi A, Sepe P, Zattarin E, et al. Predictive biomarkers of response to immunotherapy in metastatic renal cell cancer. Front Oncol. 2020;10: 1644.

54. Vano Y, Elaidi RT, Bennamoun M, et al. LBA25 Results from the phase II biomarker driven trial with nivolumab $(\mathrm{N})$ and ipilimumab or VEGFR tyrosine kinase inhibitor (TKI) in naïve metastatic kidney cancer (m-ccRCC) patients (pts): the BIONIKK trial [abstract]. Ann Oncol. 2020;31(Suppl 4): S1157.

55. McDermott DF, Huseni MA, Atkins MB, et al. Clinical activity and molecular correlates of response to atezolizumab alone or in combination with bevacizumab versus sunitinib in renal cell carcinoma. Nat Med. 2018;24(6):749-57.

56. Battle D, Bergerot CD, Msaouel P, et al. Patient preferences and expectations of systemic therapy in renal cell carcinoma [abstract]. J Clin Oncol. 2020;38(15_suppl):5083.

57. Mohamed AF, Hauber AB, Neary MP. Patient benefit-risk preferences for targeted agents in the treatment of renal cell carcinoma. Pharmacoeconomics. 2011;29(11):977-88.

58. González JM, Doan J, Gebben DJ, Boeri M, Fishman $\mathrm{M}$. Comparing the relative importance of attributes of metastatic renal cell carcinoma treatments to patients and physicians in the United States: a discrete-choice experiment. Pharmacoeconomics. 2018;36(8):973-86.

59. Mansfield C, Srinivas S, Chen C, et al. The effect of information on preferences for treatments of metastatic renal cell carcinoma. Curr Med Res Opin. 2016;32(11):1827-38.

60. World Health Organization. COVID-19 weekly epidemiological update, edition 66, 16 November 2021. 2021. https://www.who.int/publications/m/ item/weekly-epidemiological-update-on-covid19-16-november-2021. Accessed Nov 2021. 
61. Bakouny Z, Hawley JE, Choueiri TK, et al. COVID19 and cancer: current challenges and perspectives. Cancer Cell. 2020;38(5):629-46.

62. Madan A, Siglin J, Khan A. Comprehensive review of implications of COVID-19 on clinical outcomes of cancer patients and management of solid tumors during the pandemic. Cancer Med. 2020;9(24): 9205-18.

63. Abdul-Jawad S, Bau L, Alaguthurai T, et al. Acute immune signatures and their legacies in severe acute respiratory syndrome coronavirus- 2 infected cancer patients. Cancer Cell. 2021;39(2):257-75.e6.

64. Garassino MC, Whisenant JG, Huang LC, et al. COVID-19 in patients with thoracic malignancies (TERAVOLT): first results of an international, registry-based, cohort study. Lancet Oncol. 2020;21(7): 914-22.

65. Aeppli S, Eboulet EI, Eisen $\mathrm{T}$, et al. Impact of COVID-19 pandemic on treatment patterns in metastatic clear cell renal cell carcinoma. ESMO Open. 2020;5(Suppl 3):e000852.

66. Wilson J, Hakim H, Ho DY-W, et al. NCCN Best Practices Guidance: Management of COVID-19 infection in patients with cancer (March 2021). 2021. https://www.nccn.org/docs/default-source/ covid-19/2021-covid-infectious-diseasemanagement.pdf?sfvrsn=63f70c30_7. Accessed Sep 2021.

67. Curigliano G, Banerjee S, Cervantes A, et al. Managing cancer patients during the COVID-19 pandemic: an ESMO multidisciplinary expert consensus. Ann Oncol. 2020;31(10):1320-35.

68. European Society of Medical Oncology. ESMO management and treatment adapted recommendations in the COVID-19 era: renal cell cancer. 2021. https://www.esmo.org/guidelines/covid-19adapted-recommendations-slide-sets. Accessed Sep 2021. 